\title{
Research on the Cooperative Control Method of Intermittent Bus Lane and Downstream Intersection
}

\author{
Guorong Zheng (iD) and Mingyan Zhang \\ Beijing Key Lab of Urban Intelligent Traffic Control Technology, North China University of Technology, Beijing, China \\ Correspondence should be addressed to Guorong Zheng; zhengguorong@ncut.edu.cn
}

Received 20 April 2021; Revised 11 October 2021; Accepted 14 December 2021; Published 31 January 2022

Academic Editor: Lei Liu

Copyright ( 92022 Guorong Zheng and Mingyan Zhang. This is an open access article distributed under the Creative Commons Attribution License, which permits unrestricted use, distribution, and reproduction in any medium, provided the original work is properly cited.

\begin{abstract}
Intermittent bus lane (IBL) used for bus priority is a lane in which the status of a given section changes according to the presence or absence of a bus in its spatial domain. Therefore, when bus services are not so frequent, general traffic will not suffer much, and bus priority can still be obtained. In order to further explore the road resource utilization, a signal timing optimization model was proposed considering coordinate intermittent bus lanes and downstream intersections. Firstly, the functional relationship was established between the general vehicle carrying capacity and the social vehicle arrival rates, the distribution of public transportation vehicles, and the downstream intersection timing plan; then, a bilevel programming model was built which maximizes the social vehicle carrying capacity and simultaneously minimizes the delay of general vehicle intersections. Finally, the optimal solution of carrying capacity under different scenarios was analyzed through experimental simulation, which effectively reduced the delay and proved the effectiveness of the proposed method.
\end{abstract}

\section{Introduction}

In recent years, the problem of urban traffic congestion has become increasingly severe. The establishment of bus lanes is of great significance for improving the operation efficiency of public transportation, but the establishment of bus lanes has not achieved the expected results. In order to improve bus priority efficiency and road resource utilization, in 1997, Viegas and $\mathrm{Lu}$ [1] first proposed the concept of intermittent bus lane (IBL) in the traffic control system, which attracted widespread attention. Follow-up scholars conducted more in-depth research and achieved fruitful results. For the setting and implementation of bus lanes, Youbang and Li [2] conducted partial simulations on the traffic operation of intermittent bus lanes and explored the applicable conditions of the IBL strategy in terms of basic graphs, time-space graphs, average speed, and speed stability; Xianmin and Mingye [3] proposed a method for determining the clearing distance of dynamic lanes. Based on the HCM2010 vehicle delay formula and road resistance function, a model of the average and per capita travel time of vehicles under different road conditions was established, and the setting conditions of the bus lane were given; Qiufeng and Wenquan [4] analyzed the variation of the total delay error of the intersection before and after the intermittent bus entrance lane was set up and found that when the traffic saturation of the entrance lane is less than the critical traffic saturation, the delay in setting the intermittent bus-dedicated entry lane is lower; in exploring the capacity in terms of influencing factors, Yulin et al. [5] improved the space utilization rate by dynamically controlling the opening time of intermittent bus entrance lanes to social vehicles and conducted a quantitative analysis of social vehicle delays at intersections; Dingxin [6] introduced the anticollision rule which puts forward the capacity reduction mechanism of intermittent priority bus lanes and concluded that the increase in clearing distance and the decrease of the bus departure interval will reduce the capacity of intermittent priority bus lanes; Eichler and Daganzo [7] used the theory of distributed waves. The analysis found that social traffic saturation and bus departure frequency are the key factors that affect the effectiveness of intermittent bus lanes; $\mathrm{Wu}$ et al. [8] formulated mandatory 
II.SIGNAL TIMING OPTIMIZATION MODEL CONSIDERING THE INFLUENCE OF SOCIAL VEHICLES ON INTERMITTENT BUS LANES

A. Bearing Capacity Analysis for General Vehicles

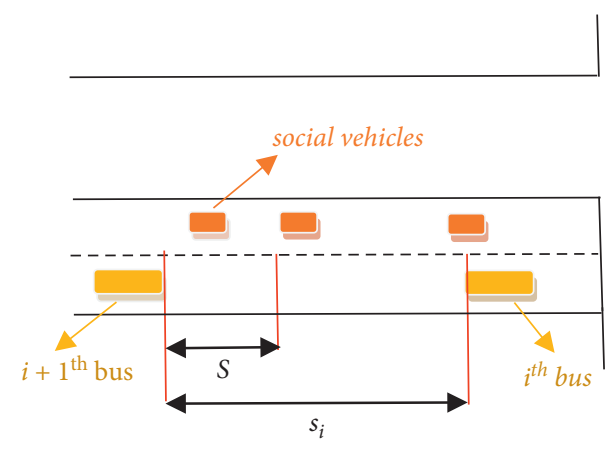

Figure 1: Bearing capacity diagram for general vehicles.

intermittent bus lane-changing rules in a vehicle-route coordination environment. The simulation model was used to analyze the control effect under different departure time intervals and vehicle density; Qiu [9] and others analyzed the impact of the IBL on the traffic density, speed, and flow of the road section through computer simulation, indicating that the service level is the turning point for the implementation of the IBL strategy; Wu et al. [10] proposed a method to provide intermittent priority for dedicated bus lanes and analyzed the influence of departure frequency on delays; in terms of signal correlation control, Guler et al. [11] proposed a similar advance signal control strategy of intermittent bus lanes which is to install auxiliary signal lights in the upstream and downstream of the main line signal to control the operation mode of the bus; $\mathrm{Ma}$ and $\mathrm{Xu}$ [12] proposed a bus priority strategy and gave a green wave coordination control method for the bus operation line; Chiabaut and Barcet [13] evaluated the impact of the intermittent bus lane strategy on traffic conditions and pointed out that the travel time of buses will be significantly reduced when combined with bus signals. In summary, the current research on intermittent bus lanes is not thorough enough. Previous studies mostly focus on the setting of bus lanes and the improvement of driving rules and less consider the impact of intermittent bus lanes on associated intersection timing. This paper considers the effect of intermittent bus lanes on the coordinated control effect of downstream intersections, optimizes the signal timing with the goal of maximum social vehicle carrying capacity and minimum delay of social vehicle intersections, and explores the changing law of the maximum carrying capacity in different scenarios based on microsimulation.

This article consists of the following parts. The first part introduces the timing optimization model under the influence of social vehicles, including the functional relationship between social vehicle carrying capacity and social vehicle arrival rate, bus distribution and downstream intersection timing plan, and the construction of a two-level programming model; the second part is the simulation analysis part, including the carrying capacity analysis under different scenarios solved by the genetic

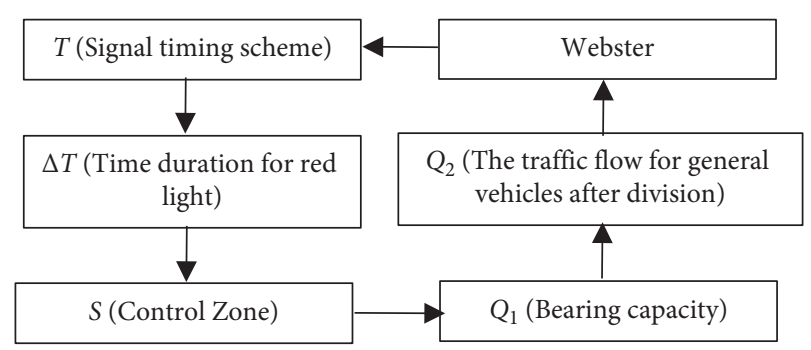

FIgURE 2: The constraint graph for the control zone of the intermittent bus lane and signal timing.

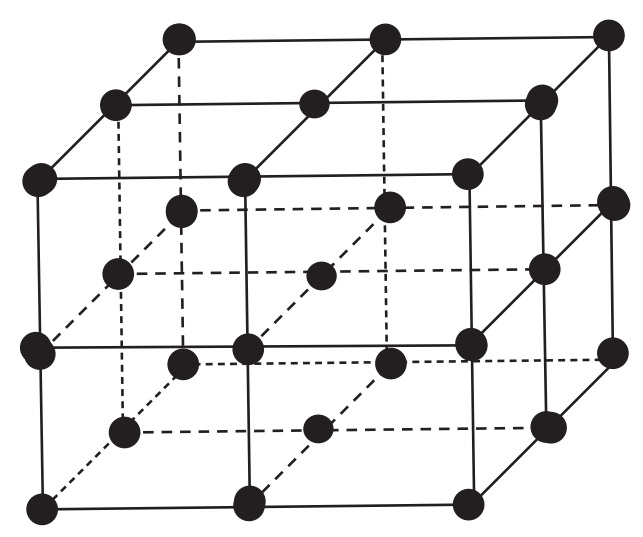

FIGURE 3: The principle for orthogonal experimental design.

algorithm; the third part summarizes the findings of this article.

\section{Signal Timing Optimization Model considering the Influence of Social Vehicles on Intermittent Bus Lanes}

2.1. Bearing Capacity Analysis for General Vehicles. As shown in Figure 1, the control zone of the intermittent bus lane will divert the traffic flow for general vehicles, which has a bearing capacity

$$
\left\{\begin{array}{l}
Q_{1}=\sum_{i}^{n-1} q_{i}, \\
q_{i}=\frac{s_{i}-S}{L} .
\end{array}\right.
$$

Among them, $n$ refers to the bus quantity within one hour, $q_{i}$ represents the maximum quantity for general vehicles that can accommodate between the $i^{\text {th }}$ and the $i+1^{\text {th }}$ bus, while $s_{i}$ refers to the space in between the $i^{\text {th }}$ and the $i+1^{\text {th }}$ bus, $S$ refers to the length of the control zone where the social vehicle cannot access, and $L$ refers to the space between vehicles.

As shown in Figure 2, the signal timing scheme for intersection will affect the length $S$ of the control zone for the intermittent bus lane, and the change of $S$ will affect the bearing capacity $Q_{1}$ for general vehicles on the mentioned lane, while the change $Q_{1}$ will have an impact on the traffic 
TABLE 1: Calculated experimental results 1 .

\begin{tabular}{lccc}
\hline$Q$ & $\mathbf{s}_{\mathbf{i}}=3 \min$ & $\mathbf{s}_{\mathbf{i}}=5 \min$ & $\mathbf{s}_{\mathbf{i}}=1 \mathrm{~min}$ \\
\hline 300 & 0.093333 & 0.12 & 0.133333 \\
600 & 0.173333 & 0.186667 & 0.193333 \\
900 & 0.168889 & 0.173333 & 0.182222 \\
1200 & 0.2 & 0.22 & 0.23 \\
1500 & 0.216 & 0.218667 & 0.234667 \\
1800 & 0.228889 & 0.251111 & 0.248889 \\
2100 & 0.224762 & 0.238095 & 0.249524 \\
2400 & 0.275 & 0.278333 & 0.281667 \\
\hline
\end{tabular}

flow $Q_{2}$ for the original lane of general vehicles so that generates influences for the timing of the intersection. So, there is a mutually restrictive relation between signal timing of downstream intersection and bearing capacity for general vehicles on the bus lane.

In order to acquire the functional relationship of bearing capacity and arrival rate for general vehicles on the bus lane and bus allocation and timing scheme for downstream intersection, the paper adopts the orthogonal experiment to optimize the execution efficiency of the algorithm.

The orthogonal experimental method is a design approach to study multiple factors and levels. It selects part of the typical points from the comprehensive experiment according to orthogonality to conduct the experiment. These typical points have features of even dispersion, neat, and comparable, as shown in Figure 3. The orthogonal experimental design is the major approach of fraction factorial design, which is an efficient, rapid, and economical experimental design method.

Various combinations of $\left(Q, s_{i}, \lambda\right)$ are, respectively, selected through the orthogonal experiment to conduct multiple simulating calculations, and $Q_{1}$ is, respectively, calculated. The combinations for $\left(Q, s_{i}, \lambda\right)$ are shown as follows:

$$
\left\{\begin{array}{l}
Q=[300,600,900,1200,1500,1800,2100,2400]\left(\frac{\mathrm{Veh}}{h}\right), \\
s_{i}=[180,300,600](s), \\
\lambda=[0.3,0.5,0.7](\%) .
\end{array}\right.
$$

Among them, $\lambda$ stands for split; the calculated experimental results are given in Tables $1-3$.

(1) When cycle $T=72 \mathrm{~s}$ and split $=36 / 36$, i.e., $\lambda=0.5$

(2) When cycle $T=72 \mathrm{~s}$ and split $=21 / 51$, i.e., $\lambda=0.3$

(3) When cycle $T=72 \mathrm{~s}$ and split $=51 / 21$, i.e., $\lambda=0.7$

As shown in Figure 4, analysis conclusions are as follows:

(1) Under the critical saturation state $(\lambda=0.5)$ and undersaturated condition, with the increase in traffic flow $Q$ for general vehicles, $Q_{1}$, the bearing capacity for general vehicles on the intermittent bus lane is in an upward trend, and compared with the undersaturated condition, the bearing capacity is more for the critical saturation state.

(2) Under the hypersaturated state $(\lambda=0.3)$, with the increase in traffic flow $Q$ for general vehicles, $Q_{1}$, the bearing capacity for general vehicles on the intermittent bus lane increases slightly, but when it exceeds the saturation flow rate under the current timing scheme, the algorithm of intermittent bus lane control fails, and $Q_{1}$ is in a downward trend.

(3) With the increase in $s_{i}$, the bus departure interval, $Q_{1}$, and the traffic flow carried on the general vehicles on the intermittent bus lane are in an upward trend.

In order to obtain the constraint relation for dependent variable $Q_{1}$ and independent variable $\left(Q, s_{i}, \lambda\right)$, the fitting function for its relevance is acquired through SPSS fitting, and the results are shown in Table 4. Among them, Beta refers to the standardization coefficient.

Then, the standardized regression equation is obtained as the following:

$$
Q_{1}=0.893 Q+0.094 s_{i}-0.204 \lambda .
$$

2.2. Two-Level Programming Model Construction. Aiming to maximize the bearing capacity for general vehicles on the intermittent bus lane and minimize the traffic delays of general vehicles at the intersection, a double-layer planning model has been established in this paper, as shown in Figure 5.

2.2.1. Upper-Layer Model. Control layer for bearing capacity of general vehicles on the bus lane:

In order to improve the utilization efficiency of the intermittent bus lane, it requires to obtain $Q_{1 \max }$ without impact on bus running.

As known from formula (1),

$$
Q_{1}=f\left(\Delta t, s_{i}\right)=\sum_{i}^{n-1} \frac{S_{i}\left(v_{2}-v_{1}-V v_{2} \Delta t\right)}{L\left(v_{2}-v_{1}\right)} .
$$

Among them, $Q_{1 \max }$ stands for the maximum bearing capacity; $s_{i}$ in formula (4) will be affected by the bus departure interval; therefore, the fixed departure interval can be treated as the known quantity; therefore, the major factor affecting $Q_{1}$ is $\Delta t$, so formula (4) can be similarly updated to

$$
\begin{aligned}
& Q_{1}=f(\Delta t), \\
& \Delta t<\frac{S_{i}\left(v_{2}-v_{1}\right)}{V v_{2}} .
\end{aligned}
$$

So, the control target for the bearing capacity for general vehicles on the bus lane on the control layer will be 

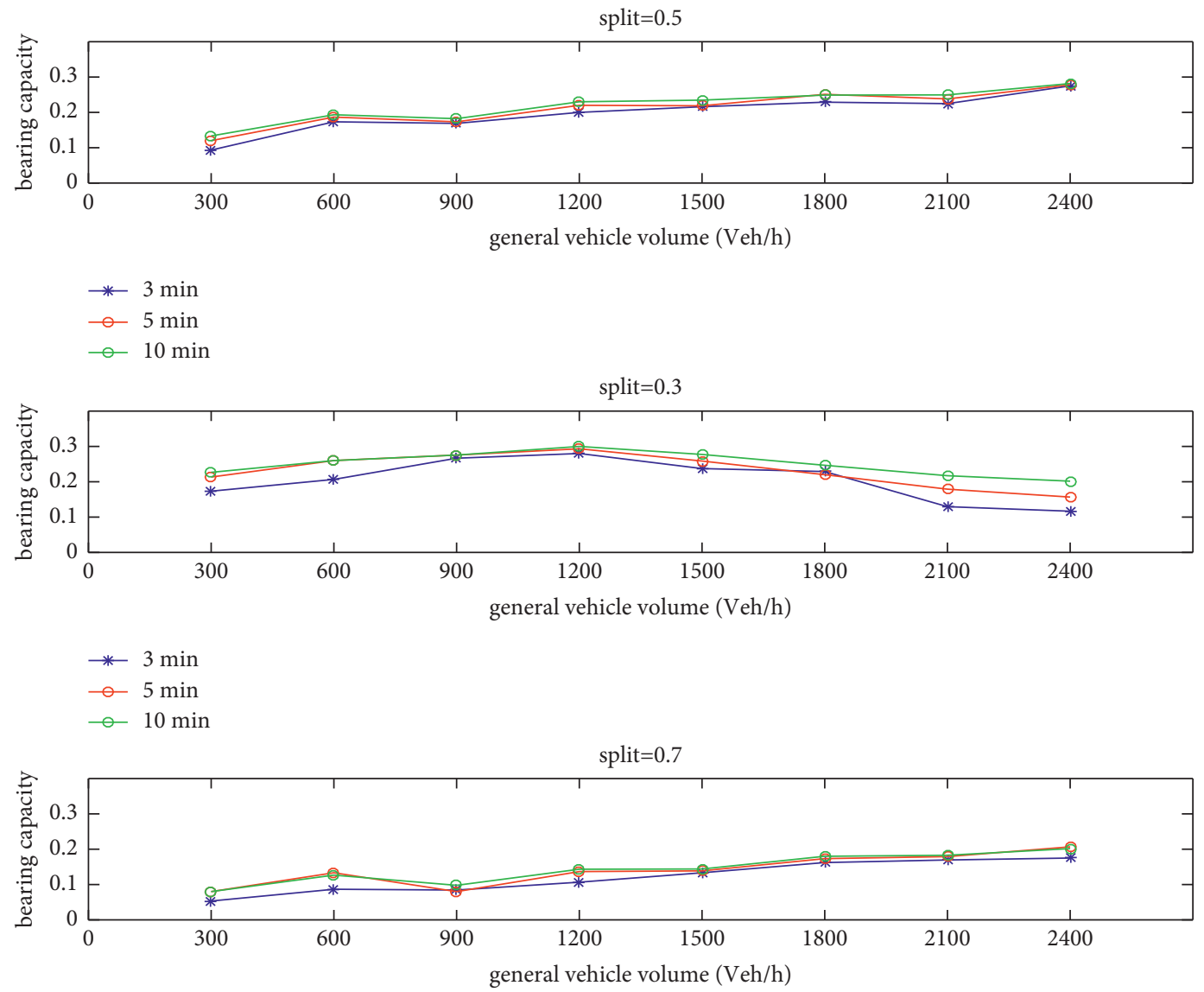

$\rightarrow 3 \mathrm{~min}$
$-\circ 5 \mathrm{~min}$
$-10 \mathrm{~min}$

Figure 4: Analysis on bearing capacity for general vehicles on the intermittent bus lane.

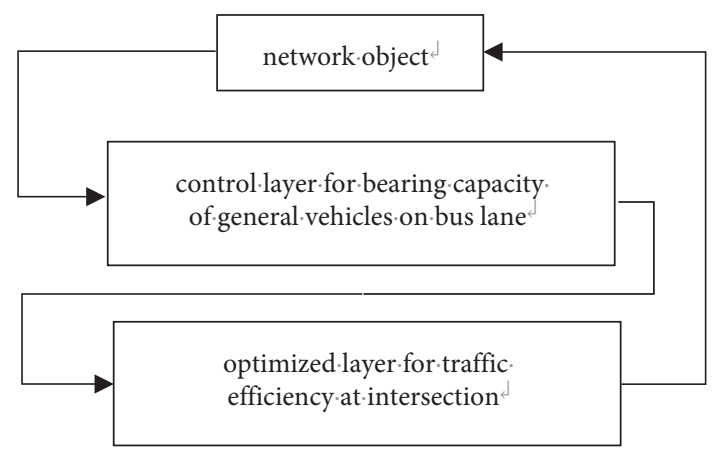

FIgURE 5: Structural diagram of the double-layer planning model.

$$
\left\{\begin{array}{l}
J_{1}=\max \left(Q_{1}\right), \\
\text { s.t. } \Delta t<\frac{S_{i}\left(v_{2}-v_{1}\right)}{V v_{2}} .
\end{array}\right.
$$

Therefore, the input of this layer will be the signal control strategy of the intersection (target-oriented time duration of red light $\Delta t$ ), and the output will be $Q_{1 \text { max }}$.
2.2.2. Lower-Layer Model. Optimized layer for traffic efficiency at the intersection:

After the traffic flow is diverted through $Q_{1 \text { max }}$, the traffic flow for general vehicles $Q_{2}=Q-Q_{1}$; at this moment, timing optimization shall be conducted according to Webster.

The bearing capacity for general vehicles on the bus lane can be improved to an extent by changing the signal strategy of downstream intersection; however, the change of split will inevitably affect the vehicle delay of that intersection; therefore, a quantitative analysis on impact degree shall be required. Currently, the access to intersection delay mainly is from the theoretical-analytical method and simulation method. The former is generally used to obtain the delay model through deduction based on definite number theory or vehicle dynamics theory. The latter extracts the relevant parameters with the help of the corresponding model established by microtraffic simulation software. In an effort to acquire detailed and comprehensive data evaluation, the simulation method is selected as the major means to evaluate the impact degree for downstream intersection.

So, the $Q_{1 \max }$ output of the upper layer will be treated as the input of this layer, and the optimized control scheme will 
TABLe 2: Calculated experimental results 2 .

\begin{tabular}{lccc}
\hline$Q$ & $S_{i}=3 \mathrm{~min}$ & $S_{i}=5 \mathrm{~min}$ & $S_{i}=1 \mathrm{~min}$ \\
\hline 300 & 0.173333 & 0.213333 & 0.226667 \\
600 & 0.206667 & 0.26 & 0.26 \\
900 & 0.266667 & 0.275556 & 0.275556 \\
1200 & 0.28 & 0.293333 & 0.3 \\
1500 & 0.237333 & 0.258667 & 0.277333 \\
1800 & 0.228889 & 0.22 & 0.246667 \\
2100 & 0.129524 & 0.179048 & 0.217143 \\
2400 & 0.116667 & 0.156667 & 0.201667 \\
\hline
\end{tabular}

TABLE 3: Calculated experimental results 3.

\begin{tabular}{lccc}
\hline$Q$ & $S_{i}=3 \mathrm{~min}$ & $S_{i}=5 \mathrm{~min}$ & $S_{i}=1 \mathrm{~min}$ \\
\hline 300 & 0.053333 & 0.08 & 0.08 \\
600 & 0.086667 & 0.133333 & 0.126667 \\
900 & 0.084444 & 0.08 & 0.097778 \\
1200 & 0.106667 & 0.136667 & 0.143333 \\
1500 & 0.133333 & 0.138667 & 0.144 \\
1800 & 0.162222 & 0.173333 & 0.18 \\
2100 & 0.169524 & 0.179048 & 0.182857 \\
2400 & 0.175 & 0.206667 & 0.201667 \\
\hline
\end{tabular}

be obtained through Webster timing algorithm with the help of the simulation model to conduct simulation analysis on the delay of general vehicles on the road section so as to estimate the pros and cons for the modified timing scheme and feedback it to the upper layer as the input.

Therefore, the objective function of this layer will be

$$
J_{2}=\min \left(h\left(Q_{1 \max }\right)\right) .
$$

Through the iterative optimization of the double-layer planning model, on the premise that without affecting the bus passage efficiency to obtain the optimized effect for the passage of general vehicles, an optimization merit function is required to be established to evaluate the results of doublelayer planning:

(1) As known from formula (4), under the perfect state, $Q_{1}$ is equal to the value when $\Delta t=0$, so make $C_{1}=J_{1} / f(0)$, which means that the larger the bearing capacity, the closer to 1 for $C_{1}$; otherwise, $C_{1}$ will be closer to 0

(2) As known from formula (7), when $Q_{1}=0$, the bearing capacity for general vehicles is 0 , and the quantity of such vehicles on the general vehicle lane is the highest, so make $C_{2}=J_{2} / h(0)$, which means that the bigger the vehicle delay, the closer to 1 for $C_{2}$; otherwise, $C_{2}$ will be closer to 0

To sum up, the optimization merit function will be

$$
C=\alpha C_{1}+\beta C_{2} .
$$

Among them, $\alpha, \beta$ refer to the weight coefficient, and $C$ refers to the evaluation index.
According to the genetic algorithm to solve $Q_{1 \text { max }}$ under the multiscene state and its corresponding intersection signal control strategy, the solving steps for the genetic algorithm shall be as follows:

Step 1: establish the optimized model and experimental calculation model.

Step 2: optimize the variable and constraint condition, the variable shall be $Q, s_{i}, \Delta t$, and $Q_{1}$, and please check constraint conditions on the preceding text.

Step 3: determine the encoding method and use the real number encoding method.

Step 4: determine the individual evaluating method. The fitness function is the objective function, which is also the acquired fitting function.

Step 5: design the genetic operator. Use the proportional selection operator for select operation, use the single-point crossover operator for crossover operation, and use the fundamental bit mutation operator for mutation operation.

Step 6: determine the operating parameter for genetic algorithm, $M$ stands for the population, $G$ refers to iterations, $P_{e}$ represents the crossover probability, and $P_{m}$ stands for the mutation probability.

\section{Simulation Verification}

After the above process, $Q_{1 \max }$ under the multiscene state and evaluation index is solved, and the simulation verification is conducted. Assuming the weight coefficient $\alpha, \beta$ are both 0.5 , the analyzed results are as the following:

(1) As shown in Table 5, when the traffic flow for general vehicles is $1500 \mathrm{Veh} / \mathrm{h}$ and the bus departure interval is 5 minutes:

Under this scene, the $2^{\text {nd }}$ scheme marked as the black border is the maximal solution of bearing capacity. The accuracy rate for the calculated value compared with the simulation value is $84.17 \%$, and the $4^{\text {th }}$ scheme marked as the black border is the optimal solution for the comprehensive evaluation index.

(2) As shown in Table 6, when the traffic flow for general vehicles is $1500 \mathrm{Veh} / \mathrm{h}$ and the bus departure interval is 10 minutes:

Under this scene, the $2^{\text {nd }}$ scheme is the maximal solution of bearing capacity. The accuracy rate for the calculated value compared with the simulation value is $87.99 \%$, and the $4^{\text {th }}$ scheme is the optimal solution for the comprehensive evaluation index.

(3) As shown in Table 7, when the traffic flow for general vehicles is $2100 \mathrm{Veh} / \mathrm{h}$ and the bus departure interval is 5 minutes: 
TABLE 4: Fitting process.

\begin{tabular}{lccccc}
\hline Model & $B$ & Standard error & Beta & $t$ & Significance \\
\hline Constant & 12.341 & 8.579 & 0 & 1.438 & 0.155 \\
$\Lambda$ & -52.604 & 12.252 & -0.204 & -4.293 & 0.000 \\
Departure interval & 0.022 & 0.011 & 0.094 & 1.985 & 0.051 \\
$Q$ & 0.055 & 0.003 & 0.893 & 18.815 & 0.000 \\
\hline
\end{tabular}

Taвle 5: General vehicles are $1500 \mathrm{Veh} / \mathrm{h}$ and the bus departure interval is 5 minutes (keep two decimal places).

\begin{tabular}{cccccccccc}
\hline No. & Split & $\begin{array}{c}\text { Calculated value for load } \\
\text { bearing ratio }\end{array}$ & $\begin{array}{c}\text { Simulation value for load } \\
\text { bearing ratio }\end{array}$ & $\begin{array}{c}\text { Bearing } \\
\text { capacity }\end{array}$ & $\begin{array}{c}\text { Average vehicle } \\
\text { delay }\end{array}$ & $c_{1}$ & $\begin{array}{c}\text { Evaluation } \\
\text { index }\end{array}$ \\
\hline 1 & 0.2 & 0.22 & 0.27 & 397.77 & 24.62 & 0.29 & 0.89 & -0.30 \\
2 & 0.3 & 0.26 & 0.31 & 460.95 & 23.98 & 0.34 & 0.87 & -0.26 \\
3 & 0.4 & 0.17 & 0.20 & 298.73 & 27.65 & 0.22 & 1.00 & -0.39 \\
4 & 0.5 & 0.22 & 0.24 & 352.77 & 17.65 & 0.26 & 0.64 & -0.18 \\
5 & 0.6 & 0.23 & 0.25 & 367.72 & 18.99 & 0.27 & 0.69 & -0.21 \\
6 & 0.7 & 0.14 & 0.17 & 248.42 & 21.65 & 0.18 & 0.78 & -0.30 \\
7 & 0.8 & 0.07 & 0.11 & 171.69 & 23.47 & 0.13 & 0.85 & -0.36 \\
\hline
\end{tabular}

TAвLE 6: General vehicles are $1500 \mathrm{Veh} / \mathrm{h}$ and the bus departure interval is 10 minutes (keep two decimal places).

\begin{tabular}{cccccccccc}
\hline No. & Split & $\begin{array}{c}\text { Calculated value for load } \\
\text { bearing ratio }\end{array}$ & $\begin{array}{c}\text { Simulation value for load } \\
\text { bearing ratio }\end{array}$ & $\begin{array}{c}\text { Bearing } \\
\text { capacity }\end{array}$ & $\begin{array}{c}\text { Average vehicle } \\
\text { delay }\end{array}$ & $c_{1}$ & $\begin{array}{c}\text { Evaluation } \\
\text { index }\end{array}$ \\
\hline 1 & 0.2 & 0.22 & 0.24 & 353.67 & 25.33 & 0.25 & 0.92 & -0.33 \\
2 & 0.3 & 0.28 & 0.32 & 472.75 & 24.12 & 0.34 & 0.87 & -0.27 \\
3 & 0.4 & 0.22 & 0.26 & 393.15 & 27.31 & 0.28 & 0.99 & -0.35 \\
4 & 0.5 & 0.23 & 0.28 & 418.78 & 18.79 & 0.30 & 0.68 & -0.19 \\
5 & 0.6 & 0.20 & 0.23 & 351.99 & 21.33 & 0.25 & 0.77 & -0.26 \\
6 & 0.7 & 0.14 & 0.17 & 248.91 & 23.33 & 0.18 & 0.84 & -0.33 \\
7 & 0.8 & 0.08 & 0.09 & 140.70 & 26.32 & 0.10 & 0.95 & -0.43 \\
\hline
\end{tabular}

TABLE 7: General vehicles are $2100 \mathrm{Veh} / \mathrm{h}$ and the bus departure interval is 5 minutes (keep two decimal places).

\begin{tabular}{ccccccccc}
\hline No. Split & $\begin{array}{c}\text { Calculated value for load } \\
\text { bearing ratio }\end{array}$ & $\begin{array}{c}\text { Simulation value for load } \\
\text { bearing ratio }\end{array}$ & $\begin{array}{c}\text { Bearing } \\
\text { capacity }\end{array}$ & $\begin{array}{c}\text { Average vehicle } \\
\text { delay }\end{array}$ & $c_{1}$ & $\begin{array}{c}\text { Evaluation } \\
\text { index }\end{array}$ \\
\hline 1 & 0.2 & 0.10 & 0.14 & 285.86 & 25.39 & 0.15 & 0.69 & -0.27 \\
2 & 0.3 & 0.18 & 0.19 & 395.76 & 25.53 & 0.21 & 0.69 & -0.24 \\
3 & 0.4 & 0.16 & 0.18 & 384.98 & 29.41 & 0.27 & 0.80 & -0.30 \\
4 & 0.5 & 0.24 & 0.24 & 505.26 & 19.15 & 0.20 & 0.52 & -0.13 \\
5 & 0.6 & 0.26 & 0.29 & 606.34 & 20.23 & 0.32 & 0.55 & -0.12 \\
6 & 0.7 & 0.18 & 0.21 & 443.30 & 23.52 & 0.23 & 0.64 & -0.20 \\
7 & 0.8 & 0.14 & 0.18 & 377.62 & 25.36 & 0.20 & 0.69 & -0.25 \\
\hline
\end{tabular}

Under this scene, the $5^{\text {th }}$ scheme is the maximal solution of bearing capacity. The accuracy rate for the calculated value compared with the simulation value is $89.76 \%$, and the $5^{\text {th }}$ scheme is the optimal solution for the comprehensive evaluation index.

\section{Conclusion}

Previous studies mostly focus on the setting of bus lanes and the improvement of driving rules and less consider the impact of intermittent bus lanes on associated intersection timing. This paper considers the effect of intermittent bus lanes on the coordinated control effect of downstream intersections.

Taking the social vehicle carrying capacity as the starting point, this paper proposes a collaborative control method for intermittent bus lanes and downstream intersections and analyzes the functional relationship between the social vehicle carrying capacity and the social vehicle arrival rate, the distribution of public transport vehicles, and the downstream intersection timing plan. A two-level planning model with the largest social vehicle carrying capacity and minimum traffic delay at social vehicle intersections is constructed, and the maximum solution of the carrying capacity in different scenarios is verified through simulation.

\section{Data Availability}

The data can be acquired by contacting the corresponding author through e-mail. 


\section{Conflicts of Interest}

The authors declare that they have no conflicts of interest.

\section{Acknowledgments}

This research was supported by the National Key Research and Development Program of China (Grant No. 2018YFB1601003) and Beijing Natural Science Foundation (Grant No. 8172018).

\section{References}

[1] J. Viegas and B. Lu, "Traffic control system with intermittent bus lanes," IFAC Proceedings Volumes, vol. 30, no. 8, pp. 865-870, 1997.

[2] Y. B. Dong, R. Li, Y. Cao, and Y. X. Chen, "Research on applicable conditions of intermittent bus lanes near stops," Journal of East China Jiaotong University, vol. 37, no. 1, pp. 132-142, 2020.

[3] X. M. Song, M. Y. Zhang, Z. J. Li, X. Wang, and Y. N. Zhang, "The setting of dynamic bus lane and its simulation analysis and evaluation," Journal of Jilin University (Engineering and Technology Edition), vol. 50, no. 5, pp. 1677-1686, 2020.

[4] Q. F. Xie, W. Q. Li, X. H. Jia, and F. Qiu, "Research on flow conditions of setting intermittent bus dedicated entrance," Journal of Transportation Engineering and Information, vol. 10, no. 2, pp. 117-124, 2012.

[5] C. Yulin, D. Yantao, and Z. Peng, "Research on optimized control system of intermittent bus entrance lane," Science Technology and Engineering, vol. 15, no. 31, pp. 96-100, 2015.

[6] W. Dingxin, Research on Capacity and Impact of Intermittent Priority Bus Lane, Southeast University, Dhaka, Bangladesh, 2019.

[7] M. Eichler, C. F. Daganzo, and Daganzo, "Bus lanes with intermittent priority: strategy formulae and an evaluation," Transportation Research Part B: Methodological, vol. 40, no. 9, pp. 731-744, 2006.

[8] D. Wu, W. Deng, Y. Song, J. Wang, and D. Kong, "Evaluating operational effects of bus lane with intermittent priority under connected vehicle environments," Discrete Dynamics in Nature and Society, vol. 2017, Article ID 1659176, 13 pages, 2017.

[9] F. Qiu, W. Li, J. Zhang, X. Zhang, and Q. Xie, "Exploring suitable traffic conditions for intermittent bus lanes," Journal of Advanced Transportation, vol. 49, no. 3, pp. 309-325, 2015.

[10] W. Wu, L. Head, S. Yan, and W. Ma, "Development and evaluation of bus lanes with intermittent and dynamic priority in connected vehicle environment," Journal of Intelligent Transportation Systems, vol. 22, no. 1-6, pp. 301-310, 2018.

[11] S. I. Guler, V. V. Gayah, and M. Menendez, "Bus priority at signalized intersections with single-lane approaches: a novel pre-signal strategy," Transportation Research Part C: Emerging Technologies, vol. 63, no. 2, pp. 51-70, 2016.

[12] C. Ma and X. D. Xu, "Providing spatial-temporal priority control strategy for brt lanes: a simulation approach," Journal of Transportation Engineering, vol. 146, no. 7, Article ID 4020060, 2020.

[13] N. Chiabaut and A. Barcet, "Demonstration and evaluation of an intermittent bus lane strategy," Public Transport, vol. 11, no. 3, pp. 443-456, 2019. 